\title{
Enhancing Drug-Drug Interaction Prediction Using Deep Attention Neural Networks
}

\author{
Shichao Liu, Member, IEEE, Yang Zhang, Yuxin Cui, Yang Qiu, Yifan Deng, Wen Zhang*, Member, \\ IEEE, Zhongfei Zhang, Fellow, IEEE
}

\begin{abstract}
Drug-drug interactions are one of the main concerns in drug discovery. Accurate prediction of drug-drug interactions plays a key role in increasing the efficiency of drug research and safety when multiple drugs are co-prescribed. With various data sources that describe the relationships and properties between drugs, the comprehensive approach that integrates multiple data sources would be considerably effective in making high-accuracy prediction. In this paper, we propose a Deep Attention Neural Network based Drug-Drug Interaction prediction framework, abbreviated as DANN-DDI, to predict unobserved drug-drug interactions First, we construct multiple drug feature networks and learn drug representations from these networks using the graph embedding method; then, we concatenate the learned drug embeddings and design an attention neural network to learn representations of drug-drug pairs; finally, we adopt a deep neural network to accurately predict drug-drug interactions. The experimental results demonstrate that our model DANN-DDI has improved prediction performance compared with state-of-the-art methods. Moreover, the proposed model can predict novel drug-drug interactions and drug-drug interaction-associated events.
\end{abstract}

Index Terms—drug-drug interactions, deep attention neural networks

\section{INTRODUCTION}

$\mathrm{D}$ RUG-DRUG Interactions (DDIs) may occur when multiple drugs are co-prescribed. Although DDIs may have beneficial effects, they sometimes have serious adverse effects and lead to drug withdrawal from the market [1]. Drug-drug interaction (DDI) prediction can help reduce the probability of adverse reactions and optimize the drug development and post-marketing surveillance process.

Clinical trials are time-consuming, expensive, and infeasible when facing the large-scale data and limitations of experimental conditions. Therefore, the researchers introduce a lot of computational methods to accelerate the prediction process. The existing computational DDI prediction methods can be roughly divided into five categories: literature extraction-based, matrix factorization-based, similaritybased, network-based and deep learning-based methods.

Literature extraction-based methods take the extraction of DDIs as a multi-class classification task, which generally extract information from informative sentences of literature, then detect candidate DDIs and classify them. Chowdhury et al. [2] proposed a multi-phase relation extraction approach, which used hybrid kernel-based support vector machine to extract the possible relationships between drugdrug pairs. Sun et al. [3] constructed a deep architecture with multiple layers of small convolutions based on a convolutional neural network to extract features automatically.

- Wen Zhang and Shichao Liu are with College of Informatics, Huazhong Agricultural University, Wuhan 430070. Email: \{zhangwen, scliu!@mail.hzau.edu.cn

- Yang Zhang, Yuxin Cui and Yang Qiu are with College of Informatics, Huazhong Agricultural University, Wuhan 430070. Email: \{tangqiaoyehua, cuiyuxin, qiuyang2000!@webmail.hzau.edu.cn

- Yifan Deng is with Electronic Information School, Wuhan University, Wuhan 430072.Email:dyfmail@whu.edu.cn

- Zhongfei Zhang is with Computer Science Department, Binghamton University, Binghamton, NY, USA. Email: zzhang@binghamton.edu

- * Corresponding author
Kim et al. [4] utilized a linear kernel-based classifier and added lexical and syntactic features to improve the accuracy of prediction. Zhang et al. [5, 6] proposed a hierarchical RNNs-based model, which combined short dependency path (SDP) and sentence sequence to obtain semantic information and used attention mechanism to map representations. Jiang et al. [7] introduced a skeleton LSTM method to effectively extract the internal structure of DDIs.

Matrix factorization-based methods decompose the drug-drug interaction matrix into several matrices, which reconstruct the interaction matrix to predict DDIs. Zhang et al. [8] introduced the manifold regularization based on drug features and proposed a novel matrix factorization method named MRMF to predict potential DDIs. Shi et al. [9] adopted the triple matrix factorization model to predict not only binary DDIs but comprehensive DDIs (i.e., enhancive and degressive DDIs) further. Yu et al. [10] designed DDINMF based on semi-nonnegative matrix factorization which decomposes the DDI adjacent matrix through the regular nonnegative matrix factorization (NMF) for enhancive and degressive DDI prediction.

Similarity-based methods assume that similar drugs could interact with the same drug. Gottlieb et al. [11] constructed a model named INDI which calculated seven types of similarity and used a weighted logistic regression classifier to predict drug-drug interactions. Cheng et al. [12] integrated a variety of drug-drug similarities to describe drugdrug pairs, and used five classifiers to build the prediction models. Foukoue et al. [13] proposed a framework named Tiresias which constructed a knowledge graph through semantic integration of data and utilized the graph to calculate similarities among drugs.

Network-based methods consider the high-order similarity of drugs and the propagation of similarity, or infer 
bioRxiv preprint doi: https://doi.org/10.1101/2021.03.16.435553; this version posted March 17, 2021. The copyright holder for this preprint (which was not certified by peer review) is the author/funder. All rights reserved. No reuse allowed without permission.

from the network structure directly. Zhang et al. [14] introduced a label propagation framework which computed the similarities of side effects, off label side effects and chemical structure, and paid attention to high-order similarity. Park et al. [15] used a random walk method to simulate signaling propagation on the protein-protein interaction (PPI) network. Lee et al. [16] constructed a heterogeneous bioinformatics network and adopted graph traversal algorithms to find paths of drug-drug pairs. Huang et al. [17] proposed a metric named S-score to measure the strength of network connections and obtained accurate prediction results through the Bayesian probability model.

Moreover, deep learning-based methods, which are specialized in extracting high-quality drug features, have been widely applied in the field of biology [18, 19] and have achieved promising results. Karim et al. [20] constructed a knowledge graph which considered combining $\mathrm{CNN}$ and LSTM model to extract local and global features of drugs through the network. Chu et al. [21] utilized a factorization auto-encoder to learn the representations of complex nonlinear drug interactions. Liu et al. [22] introduced the multimodal deep auto-encoder named DDI-MDAE based on shared latent representation to predict DDIs.

Many existing methods are proposed to predict whether drugs interact or not. In fact, diversified sub-types of interactions could instruct further research on interaction events $[23,24]$. Hence, DDI-associated events prediction has aroused some attention. Ryu et al. [25] proposed a deep learning method using structural information and a deep neural network to predict 86 important DDI types. Lee et al. [26] used auto-encoders to reduce dimensions of similarity files and applied a deep neural network to predict 106 types of DDIs. Deng et al. [27] proposed a multimodal deep learning framework which utilized multiple drug features to predict 65 categories of DDI events.

Recent studies have highlighted the importance of integrating heterogeneous drug features for the prediction of DDIs [28, 29]. However, integrating multiple diverse drug features in drug-drug interaction prediction is a challenging task. Heterogeneous drug features could be correlated and have redundant information, which may affect the performances of conventional classifiers. Hence, an effective framework of integrating heterogeneous features is in demand. Deep Neural Network (DNN) is one of the representative algorithms of deep learning, which aims to use multiple nonlinear and complex processing layers to model high-level drug features. Thus, it is a suitable model for learning high-level representations from multiple drug features.

In this paper, we propose a Deep Attention Neural Network based DDI prediction framework, abbreviated as DANN-DDI. Firstly, drug features are formulated as drug feature networks, and the graph representation learning method SDNE [30] is utilized to learn drug embeddings from these networks. Secondly, the drug embeddings are concatenated, and the attention neural network, which takes into account different contributions of different fea- tures and their dimensions, is designed to learn the representations of drug-drug pairs. Finally, the representations of drug-drug pairs are fed into a deep neural network to predict potential drug-drug interactions. The experimental results demonstrate that DANN-DDI could combine diverse drug information attentively, deliver high-accuracy performances, and outperform benchmark methods.

In summary, the main contributions of DANN-DDI are as follows: (1) We introduce a deep attention neural network framework for drug-drug interaction prediction, which can effectively integrate multiple drug features; (2) We employ the attention neural network to learn attention vectors of each of the specific drug-drug pairs; (3) Experimental results demonstrate that DANN-DDI can predict novel drug-drug interactions and DDI-associated events.

\section{MATERIALS AND METHODS}

\subsection{Dataset Description}

DrugBank [31] database integrates bioinformatics and chemical informatics resources to provide detailed drug data, including drug chemical substructures, targets, enzymes, pathways and drug-drug interactions. In this paper, we adopt the drug dataset with multiple drug features obtained from the DrugBank database released in April 2018 (version 5.1.0). Then, we use the ID mapping server to map the target proteins of the drugs into the KEGG drug database [32] for obtaining drug pathways. Our dataset contains 841 drugs with 619 chemical substructures, 1333 targets, 214 enzymes and 307 pathways, which is described in Table 1. The 841 drugs have 353,220 drug-drug pairs, including 82,620 known pairwise DDIs.

\section{TABLE 1}

THE DESCRIPTION ABOUT DRUG FEATURES AND SOURCES

\begin{tabular}{lccc}
\hline Data type & Database & Reference & Description \\
\hline Drug & DrugBank & {$[31]$} & 841 drug types \\
Target & DrugBank & {$[31]$} & 1333 target types \\
Enzyme & DrugBank & {$[31]$} & 214 enzyme type \\
Pathway & KEGG & {$[32]$} & 307 pathway types \\
Substructure & PubChem & {$[33]$} & 619 substructure types \\
\hline
\end{tabular}

\subsection{Overview of Methods}

In this paper, we propose a deep attention neural network framework named DANN-DDI to predict potential interactions between drug-drug pairs in multiple drug feature networks.

As described in Figure 1, DANN-DDI consists of three components, i.e. drug feature learning component, drugdrug pair feature learning component and drug-drug interaction prediction component. The drug feature learning component first constructs drug feature networks, and then learn the representations of drugs from these networks using graph representation learning (Figure 1a). The drugdrug pair feature learning component concatenates the five embeddings to obtain the comprehensive embeddings for drugs and designs an attention neural network to learn drug-drug pairs' representations (Figure 1b). The drug-drug 


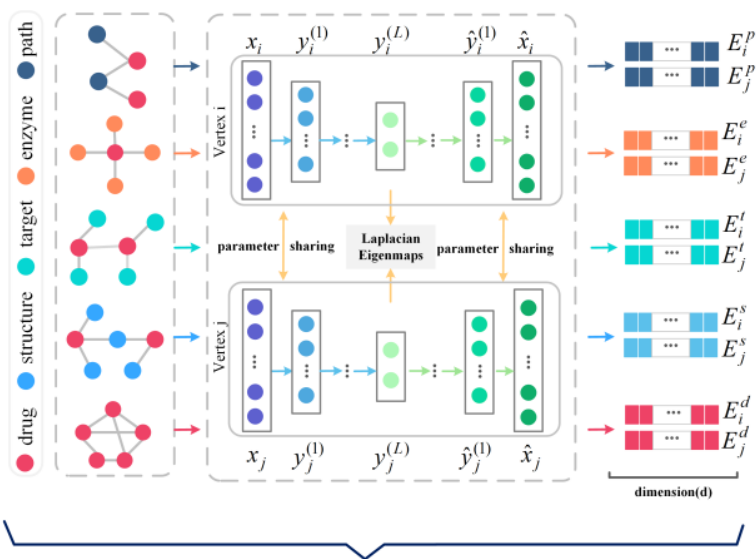

(a) Drug Feature Learning

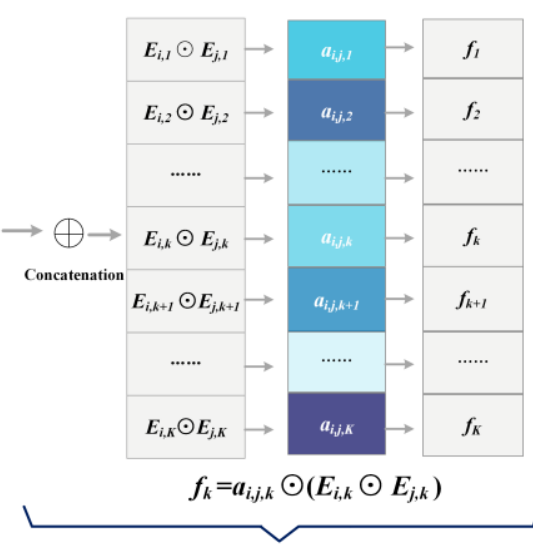

(b) Drug-drug Pair Feature Learning

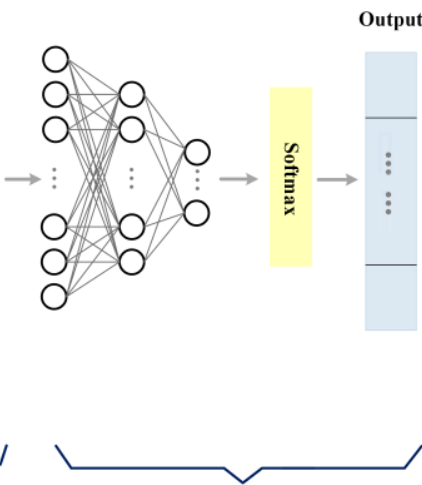

(c) Drug-drug Interaction Prediction

Fig. 1. An overview of DANN-DDI. (a) Drug Feature Learning. (b) Drug-drug pair feature learning. (c) Drug-drug interaction prediction.

interaction prediction component uses a deep neural network to predict potential drug-drug interactions, which uses the representations of drug-drug pairs as input (Figure 1c).

\subsection{Drug Feature Learning}

In this work, we collect five drug features, i.e. chemical substructures, targets, enzymes, pathways, and drug-drug interactions. The basic idea of the drug feature learning is described in Figure 1a.

\subsubsection{Construction of Drug Feature}

Due to the different effects of drug features on the performances of DDI prediction, we consider all these drug features to build five drug feature networks: drug-substructure network, drug-target network, drug-enzyme network, drug-pathway network and drug-drug interaction network. Each drug feature network includes drug nodes, feature nodes, and the links between them. The links of the networks represent associations between drugs and features. For example, a drug could have some substructures, and thus a drug node is linked to some substructure nodes in the drug-substructure network.

\subsubsection{Graph Representation Learning}

The graph embedding or representation learning is to learn the feature vectors of nodes in networks, and gains more and more attentions in bioinformatics [34]. Many graph representation learning methods have been proposed, and studies have shown that the structural deep network embedding (SDNE) could achieve competitive performances. Therefore, we adopt SDNE to learn the embeddings of drug nodes from drug feature networks.

SDNE can preserve the first-order and second-order proximity simultaneously. The first-order proximity constrains the similarity of the latent representations (i.e., $y_{i}^{(L)}$ and $y_{j}^{(L)}$ ) of a pair of vertexes (i.e., vertex $i$ and vertex $j$ ). The second-order proximity is reconstructed by minimizing the reconstruction error of the output $\hat{x}_{i}$ and the input $x_{i}$. More details can be found in [30].

We extract the representations of drug nodes in five drug feature networks. Suppose that we have $n$ drugs; the five drug embeddings from above networks are denoted as $\left\{E_{i}^{s}\right\}_{i=1}^{n},\left\{E_{i}^{t}\right\}_{i=1}^{n},\left\{E_{i}^{e}\right\}_{i=1}^{n},\left\{E_{i}^{p}\right\}_{i=1}^{n}$ and $\left\{E_{i}^{d}\right\}_{i=1}^{n}$ respectively.

\subsection{Drug-drug Pair Feature Learning}

Motivated by the successful applications of attention networks [6, 35-38], we introduce the drug-drug pair feature learning component in Figure 1b, which is an attention neural network to learn representations of drug-drug pairs by fusing learned representations of drugs from drug feature networks.

First, we concatenate the five embeddings $E_{i}^{s}, E_{i}^{t}, E_{i}^{e}$, $E_{i}^{p}$ and $E_{i}^{d}$, then obtain a comprehensive vector $E_{i}=$ $\left[E_{i}^{s}, E_{i}^{t}, E_{i}^{e}, E_{i}^{p}, E_{i}^{d}\right]$ for drug $i, i=1,2, \ldots, n$. The comprehensive vectors combine diverse information learned from drug feature networks. Let $K$ denote the dimensions of the comprehensive vectors.

Then, we learn the representations of drug-drug pairs from the comprehensive vectors of individual drugs. The representation of a drug-drug pair (drug $i$ and $\operatorname{drug} j$ ) is defined as follows:

$$
F_{i, j}=a_{i, j} \odot\left(E_{i} \odot E_{j}\right)
$$

where $\odot$ is the element-wise product. $a_{i, j}=$ $\left(a_{i, j, 1}, a_{i, j, 2}, \cdots, a_{i, j, K}\right)$ is a $K$-dimensional attention vector to capture the different importance of $K$ dimensions in $E_{i} \odot E_{j}$. Specifically, $F_{i, j}=\left(f_{1}, f_{2}, \cdots, f_{K}\right)$ and $f_{k}=a_{i, j, k} \odot\left(E_{i, k} \odot E_{j, k}\right)$. $a_{i, j, k}$ is the attention weight responsible for the $k$ th dimension, $k=1,2, \ldots, K$.

Next, we calculate the attention vector $a_{i, j}$ as follows:

$$
a_{i, j, k}=\frac{\exp \left(\hat{a}_{i, j, k}\right)}{\sum_{m=1}^{k} \exp \left(a_{i, j, m}\right)}
$$

where $\hat{a}_{i, j}=V^{T} R E L U\left(W\left[E_{i}, E_{j}\right]+b\right)$ and $R E L U$ is an activation function [39]. $b$ and $W$ are the bias vector and the weight matrix, and $V^{T}$ is the weight vector. $\left[E_{i}, E_{j}\right]$ denotes the concatenation of $E_{i}$ and $E_{j}$. Attention vector $a_{i, j, k}$ is used to capture the importance on dimension $k$ of the representation of a drug-drug pair (drug $i$ and drug $j$ ).

Therefore, the drug-drug pair feature learning component employs the attention neural network to learn representations of drug-drug pairs, which takes into account dif- 
bioRxiv preprint doi: https://doi.org/10.1101/2021.03.16.435553; this version posted March 17, 2021. The copyright holder for this preprint (which was not certified by peer review) is the author/funder. All rights reserved. No reuse allowed without permission.

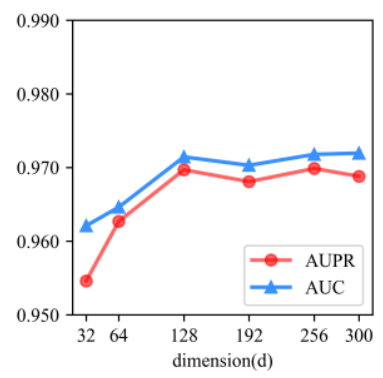

(a)

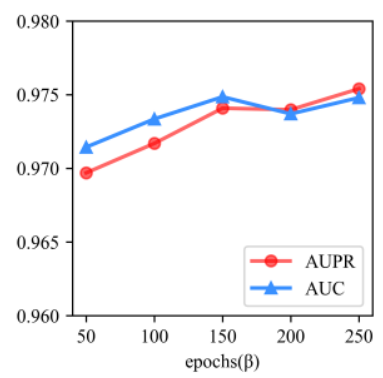

(c)

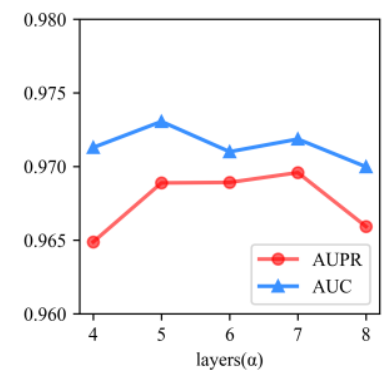

(b)

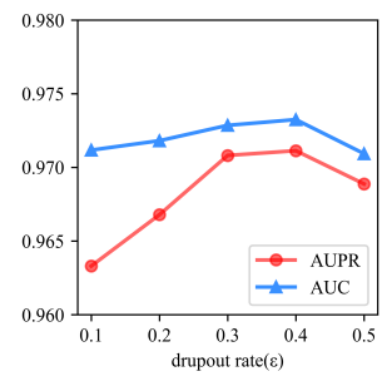

(d)

Fig. 2. Parameter Sensitivity.

ferent contributions of different features and their dimensions. The attention neural network outputs representations of drug-drug pairs.

\subsection{Drug-drug interaction prediction}

The prediction of drug-drug interactions can be considered as a binary classification problem. The drug-drug interaction prediction component uses representations of drugdrug pairs to predict potential DDIs.

The drug-drug interaction prediction component is designed as follows. As shown in Figure 1c, the input layer of the network has the same dimensions as those of the representations of drug-drug pairs. Then, the inputs pass through multiple fully connected hidden layers. The output layer contains two neurons, which denote two classes of drug-drug pairs (interaction or no-interaction). The softmax function is used for the output nodes to generate probabilities. We adopt the Rectified Linear Unit (RELU) [39] as the activation function for all the hidden layers.

\subsection{Model Optimization}

DANN-DDI consists of three components. The drug feature learning component is trained and optimized first. Then, drug-drug pair feature learning component is trained and optimized along with the drug-drug interaction prediction component in an end-to-end way.

To optimize the DANN-DDI models, we select the binary cross-entropy loss function and utilize Adam optimizer [40] with default parameters to optimize the drug-drug interaction prediction component. Between hidden layers, batch normalization layers are adopted to accelerate the convergence, and dropout layers [41] are adopted to avoid overfitting and improve generalization ability.

\section{EXPERIMENT}

\subsection{Evaluation Metrics}

In the previous work, 5-fold cross validation was adopted to evaluate prediction models $[42,43]$. In this paper, we adopt $K$-fold cross validation (i.e. $5-\mathrm{CV}$ ) to evaluate DDI prediction models. We randomly split the known drugdrug interactions into $K$ subsets with an equal size. In each fold, one subset and all unlabeled data are selected as the testing set and the rest subsets and all unlabeled data as the training set. We use the known drug-drug interactions to train the prediction model and predict unobserved interactions between drugs.

Here, several evaluation metrics are used to measure the performances of the prediction model, i.e., the area under the precision-recall curve (AUPR), the area under ROC curve (AUC), F-measure (F), the accuracy (ACC), precision and recall. In our work, known drug-drug interactions are a small proportion of all drug-drug pairs. Therefore, we take AUPR which focuses on positive examples as the primary evaluation metric.

\subsection{Parameter Discussion}

There are four key parameters: the dimension of drug embeddings $d$, the number of DNN hidden layers $\alpha$, the total epochs of DNN training $\beta$ and the dropout rate $\varepsilon$ for DNN hidden layers. Here, we discuss the influence of these parameters on the performance of DANN-DDI.

We consider the combinations of parameters: $d \in\{32,64$, $128,192,256,300\}, \alpha \in\{4,5,6,7,8\}, \beta \in\{50,100,150,200,250\}$ and $\varepsilon \in\{0,0.1,0.2,0.3,0.4,0.5\}$. We build the DANN-DDI models using different parameter combinations, and DANN-DDI models are evaluated using 3-CV. The AUPR scores (primary metric) and AUC scores are adopted to measure the performances of prediction models.

Among all parameter combinations, DANN-DDI achieves the best performance when $d=128, \alpha=7, \beta=$ 150 and $\varepsilon=0.4$. Then, we fix three parameters and discuss the influence of the remaining parameter. When fixing $\alpha, \beta$ and $\varepsilon$, the AUPR scores and AUC scores of DANN-DDI model achieve the best performances when the dimension $d$ of drug embeddings equals to 128 or 256 as shown in Figure $2 \mathrm{a}$. When fixing $d, \beta$ and $\varepsilon$, we set the number of neurons in the last hidden layer to 64 and consider the influence of $\alpha$ on DANN-DDI. The AUPR score of DANN-DDI increases as $\alpha$ increases, and it decreases after reaching the peak as given in Figure $2 b$. When $d, \alpha$ and $\varepsilon$ are fixed, Figure $2 c$ shows that the performance of DANN-DDI increases when $\beta$ increases from 50 to 150 and decreases slightly after that, and then increases when $\beta$ increases further. As shown in Figure $3 d$, when $d, \alpha$ and $\beta$ are fixed, the performance of DANN-DDI is initially improved with the increase of $\varepsilon$ varying from 0 to 0.4 and then drops after that.

We adopt $d=128, \alpha=7, \beta=150$ and $\varepsilon=0.4$ for DANN-DDI in the following studies.

\subsection{Performances of DANN-DDI}

To demonstrate good performances and robustness of DANN-DDI, we investigate three critical components of 
TABLE 2

PERFORMANCES OF DANN-DDI

\begin{tabular}{lcccccc}
\hline DRL & AUPR & AUC & F & Accuracy & Precision & Recall \\
\hline Node2vec+ANN+DNN & 0.9595 & 0.9646 & 0.9565 & 0.9947 & 0.9846 & 0.9301 \\
Metapath2vec+ANN+DNN & 0.9173 & 0.9026 & 0.8934 & 0.9874 & 0.9579 & 0.8371 \\
LINE+ANN+DNN & 0.9639 & 0.9686 & 0.9613 & 0.9952 & 0.9857 & 0.9381 \\
SDNE+ANN+DNN & $\mathbf{0 . 9 7 0 9}$ & $\mathbf{0 . 9 7 6 3}$ & $\mathbf{0 . 9 6 9 2}$ & $\mathbf{0 . 9 9 6 2}$ & $\mathbf{0 . 9 8 5 3}$ & $\mathbf{0 . 9 5 3 7}$ \\
\hline SDNE+DNN & 0.9372 & 0.9462 & 0.9305 & 0.9865 & 0.9680 & 0.8958 \\
SDNE+ANN+DNN & $\mathbf{0 . 9 7 0 9}$ & $\mathbf{0 . 9 7 6 3}$ & $\mathbf{0 . 9 6 9 2}$ & $\mathbf{0 . 9 9 6 2}$ & $\mathbf{0 . 9 8 5 3}$ & $\mathbf{0 . 9 5 3 7}$ \\
\hline SDNE+SVM & 0.4358 & 0.6972 & 0.4171 & 0.9228 & 0.3972 & 0.4390 \\
SDNE+ LR & 0.4425 & 0.7086 & 0.4217 & 0.9193 & 0.3839 & 0.4677 \\
SDNE+ RF & 0.8994 & 0.8940 & 0.8805 & 0.9864 & $\mathbf{0 . 9 9 7 3}$ & 0.7881 \\
SDNE+ DNN & $\mathbf{0 . 9 3 7 2}$ & $\mathbf{0 . 9 4 6 2}$ & $\mathbf{0 . 9 3 0 5}$ & $\mathbf{0 . 9 8 6 5}$ & 0.9680 & $\mathbf{0 . 8 9 5 8}$ \\
\hline
\end{tabular}

$D R L=$ Drug representation learning, $A N N=$ Attention neural network, $D N N=$ Deep neural networks.

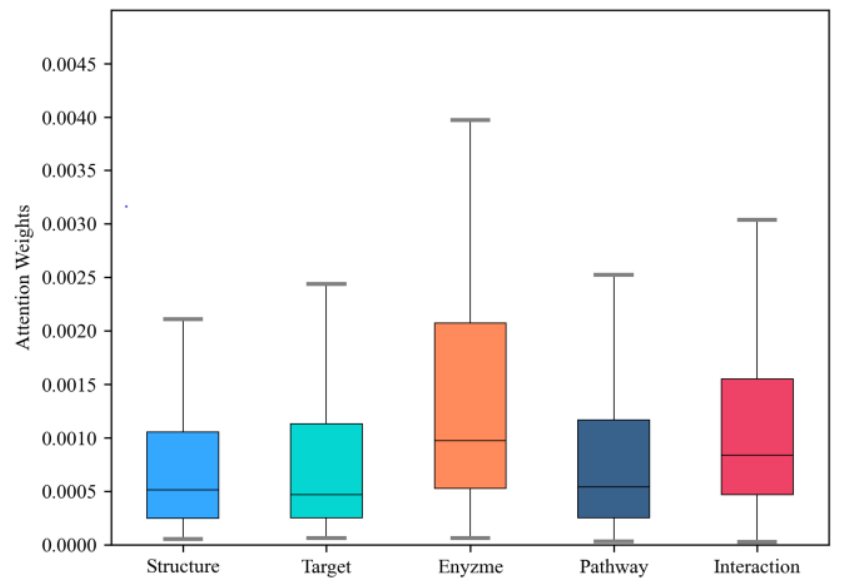

Fig. 3. Features' contributions to the prediction of drug-drug interactions. Substructure, Target, Enzyme, Pathway, and Interaction represent five drug features: chemical substructure, target, enzyme, pathway, and drug-drug interaction respectively.

DANN-DDI: drug feature learning, drug-drug pair feature learning and drug-drug interaction prediction. Here, we attempt to demonstrate that they are the leading factors for the success of DANN-DDI.

\subsubsection{Drug Feature Learning}

Drug feature learning aims to learn drug embeddings from multiple drug feature networks. Graph embedding methods in machine learning can be adopted in the drug feature learning component. We replace SDNE with several stateof-the-art graph embedding methods: Node2vec [44], Metapath2vec [45] and LINE [46]. The 5-CV results based on different graph embedding methods are reported in Table 2.

From the results, we observe that DANN-DDI using SDNE performs better than Node2vec and LINE. The representations generated by the Metapath2vec lead to the worst DDI prediction performance. The results show the effectiveness of SDNE in the DANN-DDI framework.

\subsubsection{Drug-drug Pair Feature Learning}

Drug-drug pair feature learning aims to learn effective rep- resentations for drug-drug pairs. An attention neural network is adopted in this component, which takes into account different contributions of different features and their dimensions. In order to validate the effectiveness of the attention neural network, the comparison method (SDNE+DNN) directly merge the embedding vectors of drugs to represent their pairs.

The performances of the two methods are shown in Table 2. We observe that DANN-DDI achieves a 3.60\% improvement in terms of AUPR and a 3.18\% improvement in terms of AUC over the comparison method. The results indicate that the attention neural network in DANN-DDI can efficiently learn representations of drug-drug pairs.

The representation of drug-drug pairs has the same dimensions corresponding to the representation of the drugs in pairs. To observe different contributions of the five drug features intuitively, we output the attention weights of all drug pairs from one fold in 5-CV and calculate the average weight of each dimension. The attention weights of $128 \mathrm{di}-$ mensions related to each feature are adopted, and we visualize the average weights of all drug pairs on the five features. As shown in Figure 3, we observe that the median attention weight of the enzyme is the highest, followed by the interaction, while target, structure, and pathway have lower median attention weights. In addition, enzyme and interaction features have higher attention weights on the whole. Therefore, enzyme and interaction may have more contributions to the representation of drug-drug pairs and may be more important to the prediction of drug-drug interactions.

\subsubsection{Drug-drug Interaction Prediction}

The drug-drug interaction prediction component utilizes the representations of drug-drug pairs to predict potential DDIs. In the design of DANN-DDI, we adopt the classic deep neural network (DNN) as the component. In order to test the performance of the deep neural network, we replace DNN with several classic classifiers: Random Forest (RF) [47], Support Vector Machine (SVM) [48] and Logistic Regression classifier (LR) [49]. The learned drug embeddings of the two drugs in drug-drug pairs $E_{i}$ and $E_{j}$ are directly 
bioRxiv preprint doi: https://doi.org/10.1101/2021.03.16.435553; this version posted March 17, 2021. The copyright holder for this preprint (which was not certified by peer review) is the author/funder. All rights reserved. No reuse allowed without permission.

TABLE 3

5-FOLD CROSS-VALIDATION RESULTS OF DIFFERENT MODELS

\begin{tabular}{lllllll}
\hline Method & AUPR & AUC & F & Accuracy & Precision \\
\hline PCA+NB & $0.6962+/-0.0002$ & $0.3742+/-0.0002$ & $0.2142+/-0.0001$ & $0.5058+/-0.0004$ & $0.3009+/-0.0002$ & $0.8648+/-0.0000$ \\
PCA+SVM & $0.6994+/-0.0002$ & $0.4344+/-0.0004$ & $0.3969+/-0.0003$ & $0.4395+/-0.0005$ & $0.4171+/-0.0004$ & $0.9293+/-0.0000$ \\
PCA+LR & $0.7112+/-0.0002$ & $0.4418+/-0.0003$ & $0.3849+/-0.0002$ & $0.4680+/-0.0004$ & $0.4224+/-0.0003$ & $0.9264+/-0.0000$ \\
Jaccard+NB & $0.4405+/-0.0000$ & $0.7360+/-0.0000$ & $0.1541+/-0.0000$ & $0.7102+/-0.0007$ & $0.2532+/-0.0000$ & $0.7589+/-0.0000$ \\
Jaccard+SVM & $0.6995+/-0.0001$ & $0.4347+/-0.0003$ & $0.3975+/-0.0003$ & $0.4396+/-0.0003$ & $0.4175+/-0.0003$ & $0.9294+/-0.0000$ \\
Jaccard+LR & $0.7116+/-0.0001$ & $0.4428+/-0.0002$ & $0.3864+/-0.0001$ & $0.4687+/-0.0003$ & $0.4236+/-0.0002$ & $0.9266+/-0.0000$ \\
Nearest Neighbor & $0.6344+/-0.0001$ & $0.9381+/-0.0000$ & $0.6102+/-0.0003$ & $0.9579+/-0.0002$ & $0.6601+/-0.0043$ & $0.5676+/-0.0032$ \\
Label Propagation & $0.6192+/-0.0002$ & $0.9348+/-0.0000$ & $0.6026+/-0.0003$ & $0.9567+/-0.0002$ & $0.6449+/-0.0036$ & $0.5657+/-0.0027$ \\
LPMS & $0.3826+/-0.0003$ & $0.8634+/-0.0001$ & $0.4166+/-0.0005$ & $0.9239+/-0.0011$ & $0.3758+/-0.0045$ & $0.4681+/-0.0070$ \\
MP & $0.9385+/-0.0004$ & $\mathbf{0 . 9 8 5 4 + / - 0 . 0 0 0 2}$ & $0.8922+/-0.0005$ & $0.9878+/-0.0001$ & $0.9180+/-0.0022$ & $0.8678+/-0.0019$ \\
NDLM & $0.9664+/-0.0011$ & $0.9772+/-0.0006$ & $0.9650+/-0.0011$ & $0.9960+/-0.0001$ & $0.9743+/-0.0028$ & $\mathbf{0 . 9 5 5 9 + / - 0 . 0 0 1 2}$ \\
DDI-MDAE & $0.9634+/-0.0017$ & $0.9625+/-0.0024$ & $0.9587+/-0.0021$ & $0.9926+/-0.0004$ & $\mathbf{0 . 9 9 4 5 + / - 0 . 0 0 1 6}$ & $0.9255+/-0.0048$ \\
DANN-DDI* & $0.9415+/-0.0030$ & $0.9567+/-0.0013$ & $0.9382+/-0.0029$ & $0.9924+/-0.0004$ & $0.9619+/-0.0070$ & $0.9159+/-0.0028$ \\
DANN-DDI & $\mathbf{0 . 9 7 0 9 + / - 0 . 0 0 1 9}$ & $0.9763+/-0.0010$ & $\mathbf{0 . 9 6 9 2 + / - 0 . 0 0 1 9}$ & $\mathbf{0 . 9 9 6 2 + / - 0 . 0 0 0 2}$ & $0.9853+/-0.0033$ & $0.9537+/-0.0020$ \\
\hline
\end{tabular}

concatenated as $\left[E_{i}, E_{j}\right]$. Then we feed the drug-drug pairs' representation into different classifiers to evaluate the performances.

Table 2 shows the superiority of DNN over the compared classifiers, from which DNN achieves a $4.20 \%$ improvement in terms of AUPR and a $5.84 \%$ improvement in terms of AUC compared to RF. Overall, conventional classifiers SVM and LR cannot extract high-level drug features, and results in the worst performances. The Random Forest classifier adopts the ensemble learning strategy to handle the non-linear drug feature data. Thus, it performs the second-best in terms of AUPR.

\subsection{Comparison with DDI Prediction Methods}

In this section, we compare the proposed model DANNDDI with several state-of-the-art prediction methods and baseline methods. We adjust the parameters of these comparison methods to ensure optimal performances.

\subsubsection{Compared Methods}

First, we compare the following state-of-the-art methods with DANN-DDI to evaluate the performances.

- Nearest Neighbor [50] uses the nearest neighbor similarity of drugs contained in known drug-drug interactions to predict new DDIs.

- Label Propagation [15] is a semi-supervised learning method based on graph which propagates the existing drug-drug interaction information in the network to identify novel DDIs.

- MP [43] is a matrix perturbation method assuming that random removal of a small proportion of links from the network will not alter the network structure.

- LPMS [14] is an integrative label propagation method which integrates multiple similarities derived from drug chemical substructures, drug label side effects and off-label side effects.

- NDLM [26] uses autoencoders and a deep feed-forward network that are trained using four Jaccard similarity matrixes of four drug features to predict DDIs.

- DDI-MDAE [22] utilizes multi-modal deep auto-encoders to learn unified drug representations in a shared hidden layer from multiple drug feature networks. We learn drug representations using the representation learning method in DDI-MDAE and feed them to DNN to predict DDIs.

- DANN-DDI* adopts a single heterogeneous network which integrates multiple drug feature information as the input of representation learning, and the drugdrug pair feature learning and drug-drug interaction prediction components remain unchanged.

In addition, due to the imbalanced dataset, we add simpler baseline methods to provide more comprehensive comparison. In the experiment, each drug can be represented by a binary feature vector, whose values $(1$ or 0$)$ indicate the presence or absence of the corresponding feature. Then there are two strategies: one is to utilize principal component analysis (PCA) to reduce dimensions; the other is to calculate the pairwise drug-drug similarity using Jaccard similarity measure. Then feature vectors pass through NB, SVM, and LR respectively.

\subsubsection{Cross validation results of compared methods}

To avoid the bias of results, we implement 10 runs of $5-\mathrm{CV}$ for each model respectively, and adopt the average results of the 10 runs to measure the performances.

As mentioned above, we adopt AUPR as the primary evaluation metric. As shown in Table 3, DANN-DDI outperforms all the comparison methods in most cases. DANNDDI performs slightly better than NDLM and DDI-MDAE in terms of AUPR and F-measure on 5-CV. In addition, DANN-DDI achieves a $3.45 \%$ improvement over MP in terms of AUPR and an $8.63 \%$ improvement over MP in terms of F-measure. Simple baseline methods generally report dismal results on our dataset. Moreover, standard deviation for multiple $\mathrm{CV}$ runs shows the robustness of DANN-DDI. From the overall results, we observe that our framework DANN-DDI achieves satisfactory performances in DDI prediction. 


\subsection{Case Studies}

\subsubsection{Predicting New Drug-drug interactions}

In this section, we conduct experiments to demonstrate the efficacy of DANN-DDI to predict novel DDIs.

In the experiment, the 841 drugs collected have 353,220 drug-drug pairs. Specifically, there are 82,620 known DDIs and 270,600 unlabeled pairs that may contain potential DDIs between drugs. We train DANN-DDI with all known drug-drug interactions to predict DDIs that are not contained in our datasets.

TABLE 4

TOP 20 DDIS PREDICTED BY DANN-DDI

\begin{tabular}{|c|c|c|c|}
\hline Rank & Drug 1 & Drug 2 & Evidence \\
\hline 1 & Bromocriptine & Paclitaxel & The metabolism of Paclitaxel can be decreased when combined with Bromocriptine. \\
\hline 2 & Abemaciclib & Mitotane & N.A. \\
\hline 3 & Abemaciclib & Lumacaftor & N.A. \\
\hline 4 & Amitriptyline & Mianserin & Amitriptyline may increase the anticholinergic activities of Mianserin. \\
\hline 5 & Crisaborole & Thioridazine & N.A. \\
\hline 6 & Quetiapine & Flupentixol & $\begin{array}{l}\text { The risk or severity of QTc prolongation can be increased when Flupentixol is combined with Quet- } \\
\text { iapine. }\end{array}$ \\
\hline 7 & Metamfetamine & Maprotiline & Metamfetamine may decrease the sedative and stimulatory activities of Maprotiline. \\
\hline 8 & Dextroamphetamine & Benzphetamine & $\begin{array}{l}\text { The risk or severity of serotonin syndrome can be increased when Benzphetamine is combined with } \\
\text { Dextroamphetamine. }\end{array}$ \\
\hline 9 & Rufinamide & Fluoxetine & $\begin{array}{l}\text { Rufinamide may increase the central nervous system depressant (CNS depressant) activities of } \\
\text { Fluoxetine. }\end{array}$ \\
\hline 10 & Rotigotine & Dronabinol & $\begin{array}{l}\text { Dronabinol may increase the central nervous system depressant (CNS depressant) activities of Ro- } \\
\text { tigotine. }\end{array}$ \\
\hline 11 & Bromocriptine & Nifedipine & $\begin{array}{l}\text { The risk or severity of hypotension can be increased when Bromocriptine is combined with Nifedi- } \\
\text { pine. }\end{array}$ \\
\hline 12 & Quetiapine & Pimozid & The metabolism of Quetiapine can be decreased when combined with Pimozide. \\
\hline 13 & Phentermine & Maprotiline & Phentermine may decrease the sedative and stimulatory activities of Maprotiline \\
\hline 14 & Methylergometrine & Verapamil & N.A. \\
\hline 15 & Sertraline & Isoprenaline & N.A.. \\
\hline 16 & Fluoxetine & Argatroban & The risk or severity of hemorrhage can be increased when Fluoxetine is combined with Argatroban. \\
\hline 17 & Topiroxostat & Lumacaftor & N.A. \\
\hline 18 & Rosiglitazone & Ketoprofen & $\begin{array}{l}\text { Ketoprofen may decrease the excretion rate of Rosiglitazone which could result in a higher serum } \\
\text { level. }\end{array}$ \\
\hline 19 & Rufinamide & Escitalopram & $\begin{array}{l}\text { The risk or severity of adverse effects can be increased when Rufinamide is combined with Escital- } \\
\text { opram. }\end{array}$ \\
\hline 20 & Rufinamide & Rotigotine & Rufinamide may increase the sedative activities of Rotigotine. \\
\hline
\end{tabular}

N.A.: evidence not available

Table 4 demonstrates the top 20 predicted drug-drug interactions by DANN-DDI. We search for the evidence to support the findings, and 14 novel interactions are confirmed in the 5.1.7 version of the DrugBank database (accessed on July 19, 2020). For example, it was reported that Rufinamide may increase the central nervous system depressant (CNS depressant) activities of Fluoxetine, and the studies revealed that the metabolism of Paclitaxel can be decreased when combined with Bromocriptine. The description of the interaction between Fluoxetine (DB00472) and Argatroban (DB00278) is "The risk or severity of hemorrhage can be increased when Fluoxetine is combined with Argatroban.". The case studies demonstrate the ability and power of DANN-DDI for predicting unobserved drug-drug interactions.

\subsubsection{Predicting New DDI-associated Events}

Predicting DDI-associated events is helpful to understand the underlying mechanism of adverse reactions, and to guide the combination of drugs. Hence, we extend DANNDDI to predict new DDI-associated events. The drug feature learning and drug-drug pair feature learning components of our model for DDI-associated event prediction stay the same as those for binary DDI prediction. For the settings for deep neural network, we set the number of neurons in the output layer as 65 , denoting 65 types of events. We select the softmax function to generate probabilities for the output nodes. In addition, cross-entropy loss function is adopted. We utilize the interaction event dataset by [27] and adopt the same evaluation metrics. We apply 5-fold cross-validation $(5-\mathrm{CV})$ to predict DDI-associated events between known drugs. The experimental results show that DANN- 
bioRxiv preprint doi: https://doi.org/10.1101/2021.03.16.435553; this version posted March 17, 2021. The copyright holder for this preprint (which was not certified by peer review) is the author/funder. All rights reserved. No reuse allowed without permission.

DDI can achieve an accuracy score of 0.8874 , a micro AUPR score of 0.9088 , a micro AUC score of 0.9943 and a macro-F1 score of 0.7781 . Performances of DANN-DDI are close to those of [27].

Furthermore, we conduct case studies to validate the effectiveness of our model in practice. We utilize all DDIs and their associated events in [27] to train the prediction model, and then predict associated events for other drug-drug pairs.
DDI-associated events and corresponding descriptions are illustrated in [27]. We are concerned with the ten most frequent events, numbered from \# 1 to \# 10, and examine the top 20 predictions associated with each event. We search for the evidence of the newly predicted DDI-associated events in the latest version 5.1.7 of DrugBank database.

TABLE 5

\section{CONFIRMED DDIS AND THEIR ASSOCIATED EVENTS}

\begin{tabular}{|c|c|c|}
\hline $\begin{array}{l}\text { Event } \\
\text { type }\end{array}$ & Drug names & Events \\
\hline$\# 1$ & Nateglinide and Clemastine & The metabolism of Nateglinide can be decreased when combined with Clemastine. \\
\hline$\# 2$ & Etodolac and Naproxen & The risk or severity of adverse effects can be increased when Etodolac is combined with Naproxen. \\
\hline$\# 3$ & Halofantrine and Mifepristone & The serum concentration of Halofantrine can be increased when it is combined with Mifepristone. \\
\hline$\# 4$ & $\begin{array}{l}\text { Cyproterone acetate and } \\
\text { Bosentan }\end{array}$ & The serum concentration of Cyproterone acetate can be decreased when it is combined with Bosentan. \\
\hline$\# 5$ & Homatropine and Donepezil & The therapeutic efficacy of Homatropine can be decreased when used in combination with Donepezil. \\
\hline$\# 6$ & $\begin{array}{l}\text { Buprenorphine and Hydromor- } \\
\text { phone }\end{array}$ & $\begin{array}{l}\text { Hydromorphone may increase the central nervous system depressant (CNS depressant) activities of } \\
\text { Buprenorphine. }\end{array}$ \\
\hline$\# 7$ & Amoxapine and Dronedarone & Dronedarone may increase the QTc-prolonging activities of Amoxapine. \\
\hline$\# 8$ & Bisoprolol and Diazoxide & Diazoxide may increase the hypotensive activities of Bisoprolol. \\
\hline$\# 9$ & $\begin{array}{l}\text { Carbamazepine and Droneda- } \\
\text { rone }\end{array}$ & The metabolism of Carbamazepine can be increased when combined with Dronedarone. \\
\hline$\# 10$ & Bisoprolol and Diclofenac & Diclofenac may decrease the antihypertensive activities of Bisoprolol. \\
\hline
\end{tabular}

We list ten confirmed drug-drug interaction events in Table 5. For instance, the interaction between Etodolac and Naproxen is predicted to cause event \#2. The interaction between the two drugs is described as "The risk or severity of adverse effects can be increased when Etodolac is combined with Naproxen.". The description of interaction which may lead to event \#5 between Homatropine and Donepezil is "The therapeutic efficacy of Homatropine can be decreased when used in combination with Donepezil.". The case studies demonstrate the promise of our method to detect the potential DDI-associated events.

\section{CONCLUSION}

In this paper, we study how to attentively integrate multiple drug features to predict unknown drug-drug interactions and then propose a deep attention neural network framework named DANN-DDI. We evaluate the performance of our proposed model against several state-of-the-art prediction methods and baseline methods. Extensive experimental results demonstrate the superiority of DANN-DDI compared to the comparison methods and the efficacy of the attention mechanism. More importantly, the proposed model can also find novel interactions and DDI-associated events.

In the future, we will consider optimizing and improving our work from the following aspects: (1) In the original data set, imbalanced data and the noise in the data bring challenges to the prediction accuracy. The future direction is to solve the problem of the imbalanced learning and enhance the robustness to noise; (2) Our method can be used to predict DDI-associated events, but there is still room for improvement. Some events lack detailed descriptions and proved interactions, and the interpretability of the model is needed. Enhancing the accuracy of DDI-associated event prediction can also be our future work.

\section{ACKNOWLEDGMENT}

This work was supported by the Fundamental Research Funds for the Central Universities (2662019QD011), National Key Research and Development Program of China (2018YFC1604000), the National Natural Science Foundation of China (61772381), Huazhong Agricultural University Scientific \& Technological Self- innovation Foundation. The funders have no role in study design, data collection, data analysis, data interpretation, or writing of the manuscript.

\section{APPENDIX}

The source codes of DANN-DDI are available at the following GitHub repository.

https://github.com/naodandandan/DANN-DDI

\section{REFERENCES}

[1] I. J. Onakpoya, C. J. Heneghan, and J. K. Aronson, "Postmarketing withdrawal of 462 medicinal products because of adverse drug reactions: a systematic review of the world literature," BMC medicine, vol. 14, no. 1, pp. 10, 2016.

[2] M. F. M. Chowdhury, and A. Lavelli, "FBK-irst: A multi-phase kernel based approach for drug-drug interaction detection and classification that exploits linguistic information." pp. 351-355.

[3] X. Sun, L. Ma, X. Du, J. Feng, and K. Dong, "Deep convolution neural networks for drug-drug interaction extraction." pp. $1662-1668$.

[4] S. Kim, H. Liu, L. Yeganova, and W. J. Wilbur, "Extracting drug-drug interactions from literature using a rich feature- 
bioRxiv preprint doi: https://doi.org/10.1101/2021.03.16.435553; this version posted March 17, 2021. The copyright holder for this preprint (which was not certified by peer review) is the author/funder. All rights reserved. No reuse allowed without permission.

based linear kernel approach," Journal of biomedical informatics, vol. 55, pp. 23-30, 2015

[5] Y. Zhang, W. Zheng, H. Lin, J. Wang, Z. Yang, and M. J. B. Dumontier, "Drug-drug interaction extraction via hierarchical RNNs on sequence and shortest dependency paths," vol. 34, no. 5 , pp. 828-835, 2018.

[6] H. Wang, F. Zhang, X. Xie, and M. Guo, "DKN: Deep knowledge-aware network for news recommendation." pp. 1835-1844

[7] Z. Jiang, L. Gu, and Q. Jiang, "Drug drug interaction extraction from literature using a skeleton long short term memory neural network." pp. 552-555.

[8] W. Zhang, Y. Chen, D. Li, and X. Yue, "Manifold regularized matrix factorization for drug-drug interaction prediction," Journal of biomedical informatics, vol. 88, pp. 90-97, 2018.

[9] J.-Y. Shi, H. Huang, J.-X. Li, P. Lei, Y.-N. Zhang, K. Dong, and S.-M. Yiu, "TMFUF: a triple matrix factorization-based unified framework for predicting comprehensive drug-drug interactions of new drugs," BMC bioinformatics, vol. 19, no. 14, pp. 27-37, 2018.

[10] H. Yu, K.-T. Mao, J.-Y. Shi, H. Huang, Z. Chen, K. Dong, and S.-M. J. B. s. b. Yiu, "Predicting and understanding comprehensive drug-drug interactions via semi-nonnegative matrix factorization," vol. 12, no. 1, pp. 14, 2018

[11] A. Gottlieb, G. Y. Stein, Y. Oron, E. Ruppin, and R. Sharan, "INDI: a computational framework for inferring drug interactions and their associated recommendations," Molecular systems biology, vol. 8, no. 1, 2012.

[12] F. Cheng, and Z. Zhao, "Machine learning-based prediction of drug-drug interactions by integrating drug phenotypic, therapeutic, chemical, and genomic properties," Journal of the American Medical Informatics Association, vol. 21, no. e2, pp. e278-e286, 2014

[13] A. Fokoue, M. Sadoghi, O. Hassanzadeh, and P. Zhang, "Predicting drug-drug interactions through large-scale similarity-based link prediction." pp. 774-789.

[14] P. Zhang, F. Wang, J. Hu, and R. Sorrentino, "Label propagation prediction of drug-drug interactions based on clinical side effects," Scientific reports, vol. 5, no. 1, pp. 1-10, 2015.

[15] K. Park, D. Kim, S. Ha, and D. Lee, "Predicting pharmacodynamic drug-drug interactions through signaling propagation interference on protein-protein interaction networks," PloS one, vol. 10, no. 10, 2015.

[16] K. Lee, S. Lee, M. Jeon, J. Choi, and J. Kang, "Drug-drug interaction analysis using heterogeneous biological information network." pp. 1-5.

[17] J. Huang, C. Niu, C. D. Green, L. Yang, H. Mei, and J.-D. J. Han, "Systematic prediction of pharmacodynamic drug-drug interactions through protein-protein-interaction network," PLoS computational biology, vol. 9, no. 3, 2013.

[18] H. Öztürk, A. Özgür, and E. J. B. Ozkirimli, "DeepDTA: deep drug-target binding affinity prediction," vol. 34 , no. 17, pp. i821-i829, 2018.

[19] W. Jin, K. Yang, R. Barzilay, and T. J. a. p. a. Jaakkola, "Learning multimodal graph-to-graph translation for molecular optimization," 2018.

[20] M. R. Karim, M. Cochez, J. B. Jares, M. Uddin, O. Beyan, and S. Decker, "Drug-drug interaction prediction based on knowledge graph embeddings and convolutional-LSTM network." pp. 113-123.

[21] X. Chu, Y. Lin, J. Gao, J. Wang, Y. Wang, and L. Wang, "MultiLabel Robust Factorization Autoencoder and its Application in Predicting Drug-Drug Interactions," arXiv preprint arXiv:1811.00208, 2018

[22] S. Liu, Z. Huang, Y. Qiu, Y.-P. P. Chen, and W. Zhang, "Structural Network Embedding using Multi-modal Deep Auto-encoders for Predicting Drug-drug Interactions." pp. 445-450.

[23] M. Zitnik, M. Agrawal, and J. J. B. Leskovec, "Modeling polypharmacy side effects with graph convolutional networks," vol. 34, no. 13, pp. i457-i466, 2018.

[24] W. Zheng, H. Lin, L. Luo, Z. Zhao, Z. Li, Y. Zhang, Z. Yang, and J. J. B. b. Wang, "An attention-based effective neural model for drug-drug interactions extraction," vol. 18, no. 1, pp. 445, 2017.

[25] J. Y. Ryu, H. U. Kim, and S. Y. J. P. o. t. N. A. o. S. Lee, "Deep learning improves prediction of drug-drug and drug-food interactions," vol. 115, no. 18, pp. E4304-E4311, 2018. G. Lee, C. Park, and J. J. B. b. Ahn, "Novel deep learning model for more accurate prediction of drug-drug interaction effects," vol. 20, no. 1, pp. 415, 2019.

[27] Y. Deng, X. Xu, Y. Qiu, J. Xia, W. Zhang, and S. J. B. Liu, "A multimodal deep learning framework for predicting drug-drug interaction events," 2020.

[28] D. Sridhar, S. Fakhraei, and L. J. B. Getoor, "A probabilistic approach for collective similarity-based drug-drug interaction prediction," vol. 32, no. 20, pp. 3175-3182, 2016.

[29] H. Luo, J. Wang, M. Li, J. Luo, X. Peng, F.-X. Wu, and Y. Pan "Drug repositioning based on comprehensive similarity measures and bi-random walk algorithm," Bioinformatics, vol 32, no. 17, pp. 2664-2671, 2016.

[30] D. Wang, P. Cui, and W. Zhu, "Structural deep network embedding." pp. 1225-1234.

[31] D. S. Wishart, C. Knox, A. C. Guo, S. Shrivastava, M. Hassanali, P. Stothard, Z. Chang, and J. Woolsey, "DrugBank: a comprehensive resource for in silico drug discovery and exploration," Nucleic acids research, vol. 34, no. suppl_1, pp. D668-D672, 2006.

[32] M. Kanehisa, S. Goto, M. Furumichi, M. Tanabe, and M. Hirakawa, "KEGG for representation and analysis of molecular networks involving diseases and drugs," Nucleic acids research, vol. 38, no. suppl_1, pp. D355-D360, 2010.

[33] Y. Wang, J. Xiao, T. O. Suzek, J. Zhang, J. Wang, and S. H. Bryant, "PubChem: a public information system for analyzing bioactivities of small molecules," Nucleic acids research, vol. 37, no. suppl_2, pp. W623-W633, 2009.

[34] X. Yue, Z. Wang, J. Huang, S. Parthasarathy, S Moosavinasab, Y. Huang, S. M. Lin, W. Zhang, P. Zhang, and H. J. B. Sun, "Graph embedding on biomedical networks: methods, applications and evaluations," vol. 36 , no. 4, pp. 1241-1251, 2020.

[35] Z. Cheng, Y. Ding, X. He, L. Zhu, X. Song, and M. S. Kankanhalli, "A^ 3 NCF: An Adaptive Aspect Attention Model for Rating Prediction." pp. 3748-3754.

[36] H. Wang, T. Xu, Q. Liu, D. Lian, E. Chen, D. Du, H. Wu, and W. Su, "MCNE: An End-to-End Framework for Learning Multiple Conditional Network Representations of Social Network." pp. 1064-1072.

[37] M. Qu, J. Tang, J. Shang, X. Ren, M. Zhang, and J. Han, "An attention-based collaboration framework for multi-view network representation learning." pp. 1767-1776.

[38] B. Shin, S. Park, K. Kang, and J. C. J. a. p. a. Ho, "Self-attention based molecule representation for predicting drugtarget interaction," 2019

[39] V. Nair, and G. E. Hinton, "Rectified linear units improve restricted boltzmann machines." pp. 807-814.

D. P. Kingma, and J. J. a. p. a. Ba, "Adam: A method for stochastic optimization," 2014.

[41] N. Srivastava, G. Hinton, A. Krizhevsky, I. Sutskever, and R. Salakhutdinov, "Dropout: a simple way to prevent neural networks from overfitting," The journal of machine learning research, vol. 15, no. 1, pp. 1929-1958, 2014.

[42] W. Zhang, K. Jing, F. Huang, Y. Chen, B. Li, J. Li, and J. Gong, "SFLLN: a sparse feature learning ensemble method with linear neighborhood regularization for predicting drug-drug interactions," Information Sciences, vol. 497, pp. 189-201, 2019.

[43] W. Zhang, Y. Chen, F. Liu, F. Luo, G. Tian, and X. Li, "Predicting potential drug-drug interactions by integrating chemical, biological, phenotypic and network data," BMC bioinformatics, vol. 18, no. 1, pp. 18, 2017.

[44] A. Grover, and J. Leskovec, "node2vec: Scalable feature learning for networks." pp. 855-864.

[45] Y. Dong, N. V. Chawla, and A. Swami, "metapath2vec: Scalable representation learning for heterogeneous networks." pp. $135-144$

[46] J. Tang, M. Qu, M. Wang, M. Zhang, J. Yan, and Q. Mei, "Line: Large-scale information network embedding." pp. 1067-1077. M. Pal, "Random forest classifier for remote sensing classification," International Journal of Remote Sensing, vol. 26, no. 1, pp. 217-222, 2005 
bioRxiv preprint doi: https://doi.org/10.1101/2021.03.16.435553; this version posted March 17, 2021. The copyright holder for this preprint (which was not certified by peer review) is the author/funder. All rights reserved. No reuse allowed without permission.

[48] T. Joachims, "Training linear SVMs in linear time." pp. $217-$ 226.

[49] R. Detrano, A. Janosi, W. Steinbrunn, M. Pfisterer, J.-J. Schmid, S. Sandhu, K. H. Guppy, S. Lee, and V. Froelicher, "International application of a new probability algorithm for the diagnosis of coronary artery disease," The American journal of cardiology, vol. 64, no. 5, pp. 304-310, 1989.

[50] S. Vilar, R. Harpaz, E. Uriarte, L. Santana, R. Rabadan, and C. Friedman, "Drug-drug interaction through molecular structure similarity analysis," Journal of the American Medical Informatics Association, vol. 19, no. 6, pp. 1066-1074, 2012. 


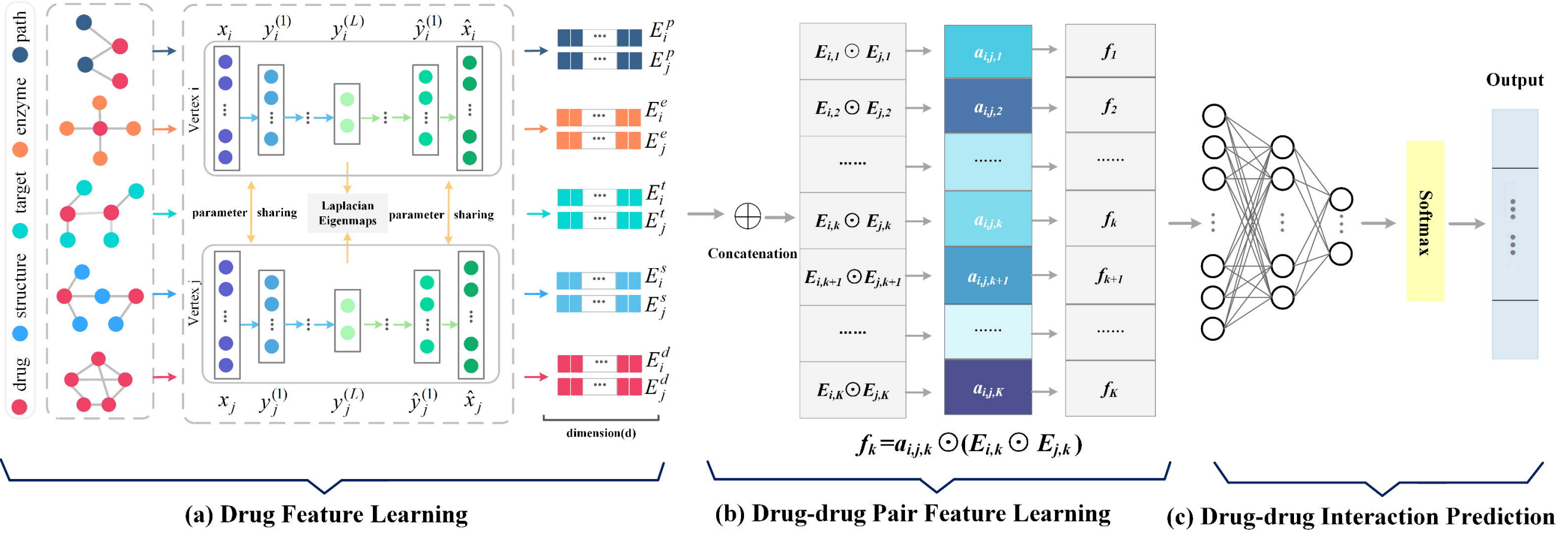




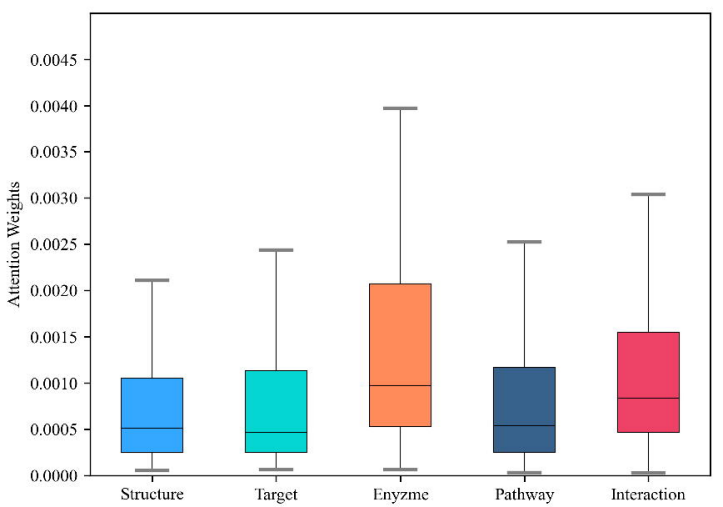

